\title{
Incomparable copies of a poset in the Boolean lattice
}

\author{
Gyula O.H. Katona* and Dániel T. Nagy ${ }^{\dagger}$
}

August 2, 2014

\begin{abstract}
Let $B_{n}$ be the poset generated by the subsets of $[n]$ with the inclusion as relation and let $P$ be a finite poset. We want to embed $P$ into $B_{n}$ as many times as possible such that the subsets in different copies are incomparable. The maximum number of such embeddings is asymptotically determined for all finite posets $P$ as $\frac{1}{t(P)}\left(\begin{array}{c}n \\ \lfloor n / 2\rfloor\end{array}\right)$, where $t(P)$ denotes the minimal size of the convex hull of a copy of $P$. We discuss both weak and strong (induced) embeddings.
\end{abstract}

\section{Introduction}

The problem discussed here is motivated by the problem of determining the largest families in $B_{n}$ avoiding certain configurations of inclusion.

Definition Let $B_{n}$ be the Boolean lattice, the poset generated by the subsets of $[n]=\{1,2, \ldots, n\}$ with the inclusion as relation and $P$ be a finite poset with the relation $<_{p}$. (If $S$ is a set of size $n$ we may also write $\left.B_{S}.\right) f: P \rightarrow B_{n}$ is an embedding of $P$ into $B_{n}$ if it is an injective function that satisfies $f(a) \subset f(b)$ for all $a<_{p} b . f$ is called an induced embedding if it is an injective function such that $f(a) \subset f(b)$ if and only if $a<_{p} b . f(P) \subseteq B_{n}$ is called a copy of $P$ in $B_{n}$.

Definition Let $X$ and $Y$ be two sets of subsets of $[n] . X$ and $Y$ are incomparable if there are no sets $x \in X$ and $y \in Y$ such that $x \subseteq y$ or $y \subseteq x$. A family $\mathcal{S}$ of sets of subsets of $[n]$ is an incomparable family if the sets of subsets in it are pairwise incomparable ( $x \nsubseteq y$ for all $x \in X, y \in Y, X \neq Y, X, Y \in \mathcal{S})$.

Definition Let $P_{1}, P_{2}, \ldots P_{k}$ be finite posets. $\operatorname{La}\left(n,\left\{P_{1}, \ldots P_{k}\right\}\right)$ denotes the size of the largest subset $\mathcal{F} \subset B_{n}$ such that none of the posets $P_{i}$ can be embedded into $\mathcal{F}$.

The value of $\operatorname{La}(n, P)$ is not known for a general poset $P$, but many special cases have been solved. See [2] for posets whose Hasse diagram is a tree. See [6] for chain posets, [7] for diamond and harp posets, [11] for improved results about the 2-diamond, and [8] for batons and certain 2-level posets. [3] provides upper bounds on $\mathrm{La}(n, P)$ for all posets $P$.

*Alfréd Rényi Institute of Mathematics

${ }^{\dagger}$ Eötvös Loránd University, Budapest 
Let $V_{k}$ denote the $(k+1)$-element poset that has a minimal element contained in the other $k$ unrelated elements. $\Lambda_{k}$ is obtained from $V_{k}$ by reversing the relations. Katona and Tarján proved that a subset of $B_{n}$ containing none of the posets $\left\{V_{2}, \Lambda_{2}\right\}$ has at most $\left(\begin{array}{c}n-1 \\ \left\lfloor\frac{n-1}{2}\right\rfloor\end{array}\right)$ elements, and this bound is sharp [10]. Such a family consists of pairwise incomparable copies of the one-element poset and the two-element chain.

In the above example, we have seen that a family not containing certain posets necessarily consists of pairwise incomparable copies of some other posets. Another example of this is determining $\operatorname{La}\left(n, V_{2}\right)$. (See [4] for asymptotic bounds on $\mathrm{La}\left(n, V_{r}\right)$.) A $V_{2}$-free family consists of pairwise independent copies of the posets $\left\{\Lambda_{0}, \Lambda_{1}, \Lambda_{2}, \ldots\right\}$. Such examples motivate the study of families of subsets consisting of pairwise independent copies of some fixed posets. In the present paper we consider the case when all the incomparable components are the copies of a single fixed poset $P$.

We investigate the following problem. How many times can we embed a poset into $B_{n}$ such that the resulting copies form an incomparable family? An asymptotic answer is given in both the induced and the non-induced case. Before we can state our main result, some notations are needed.

Notation Let $F \subseteq B_{n}$. The convex hull of $F$ is the set

$$
\operatorname{conv}(F)=\left\{b \in B_{n} \mid \exists a, c \in F \quad a \subseteq b \subseteq c\right\} .
$$

We use the following notations for the minimal size of the convex hull. For a finite poset $P$

$$
\begin{aligned}
& t_{1}(P)=\min _{f, n}\left\{|\operatorname{conv}(f(P))| \mid f: P \rightarrow B_{n} \text { is an embedding }\right\} \\
& t_{2}(P)=\min _{f, n}\left\{|\operatorname{conv}(f(P))| \mid f: P \rightarrow B_{n} \text { is an induced embedding }\right\}
\end{aligned}
$$

Note that we have to minimize over both $f$ and $n$, since if we want to embed $P$ into a lattice $B_{n}$ with larger $n$, then there might be an embedding that produces a copy with smaller convex hull.

Theorem 1.1. Let $P$ be a finite poset. Let $M_{1}(P, n)$ (and $M_{2}(P, n)$ ) denote the largest $M$ such that there are embeddings (induced embeddings) $f_{1}, f_{2}, \ldots f_{M}: P \rightarrow B_{n}$ such that $\left\{f_{i}(P), i=1,2, \ldots M\right\}$ is an incomparable family. Then

$$
\begin{aligned}
& \lim _{n \rightarrow \infty} \frac{M_{1}(P, n)}{\left(\begin{array}{c}
n \\
\lfloor n / 2\rfloor
\end{array}\right)}=\frac{1}{t_{1}(P)} \\
& \lim _{n \rightarrow \infty} \frac{M_{2}(P, n)}{\left(\begin{array}{c}
n \\
\lfloor n / 2\rfloor
\end{array}\right)}=\frac{1}{t_{2}(P)} .
\end{aligned}
$$

We prove upper and lower bounds for $M_{j}(P, n)$ in the next two sections (Theorem 2.2 and Theorem 3.3). The two bounds will imply the theorem immediately. Since the proofs are almost identical for $j=1,2$, they will be done simultaneously.

Remark Theorem 1.1. was independently proved by A. P. Dove and J. R. Griggs [5].

\section{The upper bound}

To prove the upper bound for $M_{j}(P, n)$ we need a lemma about full chains. Let $S$ be a set of size $n$. A full chain in $S$ is a set of subsets $\emptyset=C_{0} \subset C_{1} \subset C_{2} \subset \cdots \subset C_{n}=S$, where $\left|C_{m}\right|=m$ for all $m=0,1, \ldots, n$. 
Lemma 2.1. Let $\mathcal{F}$ be a family of subsets of $S$, where $|S|=n$ and $|\mathcal{F}|=t$. Then the number of full chains intersecting at least one member of $\mathcal{F}$ is at least

$$
\left(t-\frac{t(t-1)}{n}\right)\lfloor n / 2\rfloor !\lceil n / 2\rceil ! \text {. }
$$

Proof. We prove the lemma by induction on $t$. The statement is true for $t=1$, as the number of full chains passing through a subset $F$ is $|F| !(n-|F|) ! \geq\lfloor n / 2\rfloor !\lceil n / 2\rceil !$. Now let $t \geq 2$, and $\mathcal{F}=\left\{F_{1}, F_{2}, \ldots F_{t}\right\}$. Since taking complements does not change the number of intersecting chains, we may assume that some set of $\mathcal{F}$ has size at most $\lfloor n / 2\rfloor$. We can also assume that $F_{t}$ is one of the smallest subsets.

By induction, the number of full chains intersecting $\mathcal{F} \backslash\left\{F_{t}\right\}$ is at least

$$
\left(t-1-\frac{(t-1)(t-2)}{n}\right)\lfloor n / 2\rfloor !\lceil n / 2\rceil ! \text {. }
$$

The number of full chains through $F_{t}$ is $\left|F_{t}\right| !\left(n-\left|F_{t}\right|\right)$ !. Assume that $F_{t} \subset F_{i}$ for some $i \in[1, t-1]$. The number of full chains intersecting both $F_{t}$ and $F_{i}$ is $\left|F_{t}\right| !\left(\left|F_{i}\right|-\left|F_{t}\right|\right) !\left(n-\left|F_{i}\right|\right) ! \leq\left|F_{t}\right| !\left(n-\left|F_{t}\right|-1\right)$ !. Take all the full chains passing through $F_{t}$, then remove the ones passing through any of the sets $F_{1}, F_{2}, \ldots, F_{t-1}$. The remaining full chains intersect $\mathcal{F}$ only in $F_{t}$, and their number is at least

$$
\left|F_{t}\right| !\left(n-\left|F_{t}\right|\right) !-(t-1)\left|F_{t}\right| !\left(n-\left|F_{t}\right|-1\right) !=\left|F_{t}\right| !\left(n-\left|F_{t}\right|\right) !\left(1-\frac{t-1}{n-\left|F_{t}\right|}\right) \geq\lfloor n / 2\rfloor !\lceil n / 2\rceil !\left(1-\frac{2(t-1)}{n}\right) .
$$

The statement of the lemma follows after summation:

$$
\left(t-1-\frac{(t-1)(t-2)}{n}\right)+\left(1-\frac{2(t-1)}{n}\right)=t-\frac{t(t-1)}{n} .
$$

Theorem 2.2. For any finite poset $P$

$$
M_{j}(P, n) \leq \frac{1}{t_{j}(P)}\left(\begin{array}{c}
n \\
\lfloor n / 2\rfloor
\end{array}\right)\left(1+O\left(n^{-1}\right)\right)
$$

holds for $j=1,2$.

Proof. Assume that $f_{1}, f_{2}, \ldots f_{k}: P \rightarrow B_{n}$ are embeddings (induced if $j=2$ ) such that $\left\{f_{i}(P), i=\right.$ $1,2, \ldots k\}$ is an incomparable family. Then $\left\{\operatorname{conv}\left(f_{i}(P)\right), i=1,2, \ldots k\right\}$ is also an incomparable family. To see that, assume there are sets $a, b$ such that $a \subseteq b, a \in \operatorname{conv}\left(f_{i}(P)\right), b \in \operatorname{conv}\left(f_{j}(P)\right)$ and $i \neq j$. Then by the definition of the convex hull there are sets $a^{\prime} \in f_{i}(P)$ and $b^{\prime} \in f_{j}(P)$ such that $a^{\prime} \subseteq a \subseteq b \subseteq b^{\prime}$. But $a^{\prime} \nsubseteq b^{\prime}$ since $\left\{f_{i}(P), i=1,2, \ldots k\right\}$ is an incomparable family.

Since the $\left\{\operatorname{conv}\left(f_{i}(P)\right), i=1,2, \ldots k\right\}$ is an incomparable family, every full chain intersects at most one of its members. $\left|\operatorname{conv}\left(f_{i}(P)\right)\right| \geq t_{j}(P)$ for all $i$ by the definition of $t_{j}(P)$. By Lemma 2.1., each $\operatorname{conv}\left(f_{i}(P)\right)$ intersects at least $t_{j}(P)\lfloor n / 2\rfloor !\lceil n / 2\rceil !\left(1-O\left(n^{-1}\right)\right)$ chains. Since the total number of full chains is $n !$,

$$
k \leq \frac{n !}{t_{j}(P)\lfloor n / 2\rfloor !\lceil n / 2\rceil !\left(1-O\left(n^{-1}\right)\right)}=\frac{1}{t_{j}(P)}\left(\begin{array}{c}
n \\
\lfloor n / 2\rfloor
\end{array}\right)\left(1+O\left(n^{-1}\right)\right) .
$$




\section{The lower bound}

In this section our aim is to prove a lower bound on $M_{j}(P, n)$ by embedding many copies of $P$ to $B_{n}$. We need the following lemmas for the construction.

Lemma 3.1. Let $P$ be a finite poset, and let $f: P \rightarrow B_{m}$ be an embedding. Then we can label the elements of $B_{m}$ with the numbers $1,2, \ldots 2^{m}$ such that each set gets a higher number than any of its subsets, and the numbers assigned to the elements of conv $(f(P))$ form an interval in $\left[1,2^{m}\right]$.

Proof. We divide the elements of $B_{m}$ into three pairwise disjoint groups:

$$
\begin{aligned}
& \text { Let } \mathcal{F}_{1}=\left\{b \in B_{m} \mid \exists c \in f(P) \quad b \subseteq c, \nexists a \in f(P) \quad a \subseteq b\right\}, \\
& \mathcal{F}_{2}=\operatorname{conv}(f(P))=\left\{b \in B_{m} \mid \exists a, c \in f(P) \quad a \subseteq b \subseteq c\right\} \\
& \text { and } \mathcal{F}_{3}=B_{m} \backslash\left(\mathcal{F}_{1} \cup \mathcal{F}_{2}\right)=\left\{b \in B_{m} \mid \nexists c \in f(P) \quad b \subseteq c\right\} .
\end{aligned}
$$

We use the numbers of $\left[1,\left|\mathcal{F}_{1}\right|\right]$ for the sets of $\mathcal{F}_{1}$, the numbers of $\left[\left|\mathcal{F}_{1}\right|+1,\left|\mathcal{F}_{1}\right|+\left|\mathcal{F}_{2}\right|\right]$ for the sets of $\mathcal{F}_{2}$ and the numbers $\left[\left|\mathcal{F}_{1}\right|+\left|\mathcal{F}_{2}\right|+1,2^{m}\right]$ for the sets of $\mathcal{F}_{3}$. In the groups we assign numbers such that the elements representing larger subsets get larger numbers.

We have to check that if $x, y \in B_{m}$ and $y$ got a larger number than $x$, then $y \not \subset x$.

If $x$ and $y$ are in the same group, then $|x| \leq|y|$, so $y \not \subset x$. If $x \in \mathcal{F}_{1}$ and $y \in \mathcal{F}_{2}$, then $y \not \subset x$, because $y$ contains an element of $f(P)$ while $x$ does not. If $x \in \mathcal{F}_{1} \cup \mathcal{F}_{2}$ and $y \in \mathcal{F}_{3}$, then $y \not \subset x$, because $x$ is the subset of an element of $f(P)$ while $y$ is not.

Lemma 3.2. Let $P$ be a finite poset and let $\varepsilon^{\prime}>0$ be fixed. Let $j \in\{1,2\}$. Then there are integers $N, K$ and functions $f_{1}, f_{2}, \ldots f_{K}: P \rightarrow B_{N}$ such that

(i) For all $i \in[1, K], f_{i}$ is an embedding if $j=1$, and an induced embedding if $j=2$.

(ii) $K \geq \frac{2^{N}\left(1-\varepsilon^{\prime}\right)}{t_{j}(P)}$.

(iii) If $i_{1}<i_{2}, A \in f_{i_{1}}(P)$ and $B \in f_{i_{2}}(P)$, then $B \nsubseteq A$.

Proof. Let $P$ be a fixed finite poset. There is embedding (or induced embedding, if $j=2$ ) $f: P \rightarrow B_{m}$ for some $m$ such that $|\operatorname{conv}(f(P))|=t_{j}(P)$. Fix $m$ and $f$. Choose $k \in \mathbb{N}$ such that $\left(1-\frac{t_{j}(P)}{2^{m}}\right)^{k} \leq \varepsilon^{\prime}$, and let $N=k m$. Let $S_{1}, S_{2}, \ldots S_{k}$ be pairwise disjoint sets of size $m$ and let $S=\bigcup_{i=1}^{k} S_{i}$. Consider the elements of $B_{N}$ as the subsets of $S$.

Let $g_{i}: P \rightarrow B_{S_{i}}(i=1,2, \ldots k)$ be embeddings mapping $P$ into the copies of $B_{m}$ the same way as $f$ does. Assign the numbers $1,2, \ldots, 2^{m}$ to the subsets of $S_{i}$ as in Lemma 3.1. For all $i$, the elements of $\operatorname{conv}\left(g_{i}(P)\right)$ will get the numbers of the interval $I=\left[p, p+t_{j}(P)-1\right]$ for some fixed integer $p$.

In the next paragraph we will define some embeddings called good embeddings. Later we assign the names $f_{1}, f_{2}, \ldots, f_{K}$ to them and show that they satisfy the conditions $(i)-(i i i)$. Before stating the formal definition, let us explain how the good embeddings look like. A good embedding maps the elements of $P$ to subsets of the set $S=\bigcup_{i=1}^{k} S_{i}$. We define such an embedding $g: P \rightarrow B_{S}$ by determining $x \cap S_{i}$ for all $x \in P$ and $i=1,2, \ldots, k$. The good embedding will map the elements like $f$ does in the case of $S_{i}$, 
but maps all the elements of $P$ the the same set $A_{j} \subseteq S_{j}$ for $j \neq i$. We also need that $A_{j}$ is a set whose label is not in the interval $I$.

We call an embedding $g: P \rightarrow B_{S}$ good if there is an index $i \in[1, k]$ and there are $k-1$ sets $A_{1} \subseteq S_{1}, A_{2} \subseteq S_{2}, \ldots, A_{i-1} \subseteq S_{i-1}, A_{i+1} \subseteq S_{i+1}, \ldots, A_{k} \subseteq S_{k}$ such that none of the numbers assigned to $A_{1}, A_{2}, \ldots, A_{i-1}$ is in $I$, and for any $x \in P, g(x) \cap S_{i}=g_{i}(x)$, and $g(x) \cap\left(S \backslash S_{i}\right)=\bigcup_{r \in[n] \backslash\{i\}} A_{r}$.

Since the good embeddings are defined by the choice of the sets $A_{1}, \ldots, A_{i-1}$ and $A_{i+1}, \ldots, A_{k}$, the number of good embeddings is

$$
\begin{gathered}
K=\sum_{i=1}^{k}\left(2^{m}-t_{j}(P)\right)^{i-1} \cdot\left(2^{m}\right)^{k-i}=2^{N-m} \sum_{i=1}^{k}\left(1-\frac{t_{j}(P)}{2^{m}}\right)^{i-1}= \\
2^{N-m} \frac{1-\left(1-\frac{t_{j}(P)}{2^{m}}\right)^{k}}{\frac{t_{j}(P)}{2^{m}}}=\frac{2^{N}}{t_{j}(P)}\left(1-\left(1-\frac{t_{j}(P)}{2^{m}}\right)^{k}\right) \geq \frac{2^{N}\left(1-\varepsilon^{\prime}\right)}{t_{j}(P)} .
\end{gathered}
$$

The above inequality holds because we fixed $k$ to be large enough to satisfy it in the beginning of the proof.

Let $f_{1}, f_{2}, \ldots, f_{K}$ be the good embeddings. They are embeddings (induced if $j=2$ ), and their number is sufficiently large. So $(i)$ and $(i i)$ are satisfied. Now we find an ordering and reassign the names $f_{1}, f_{2}, \ldots$ to the good embeddings such that $(i i i)$ is satisfied.

Let $g$ be the good embedding defined by the index $i$ and the subsets $A_{1}, A_{2}, \ldots, A_{i-1}, A_{i+1}, \ldots, A_{k}$. Define the code of $g$ as a vector of length $k$ with coordinates as follows. The $i$ th coordinate is $p$, the smallest number in $I$. For $1 \leq j \leq k, j \neq i$, the $j$ th coordinate is the number assigned to $A_{j}$. Now take the lexicographic ordering of these codes, and reassign the names $f_{1}, f_{2}, \ldots$ to the good embeddings according to the ordering. ( $f_{1}$ will be the good embedding whose code comes first in the lexicographic ordering, $f_{2}$ will be the second and so on.)

Now we can verify (iii). Assume that $A \in f_{i_{1}}(P), B \in f_{i_{2}}(P), i_{1}<i_{2}$ and $B \subseteq A$. Let the $l$ th be the first coordinate where the codes of $f_{i_{1}}$ and $f_{i_{2}}$ are different. Since $i_{1}<i_{2}$, the $l$ th coordinate of $f_{i_{2}}$ is strictly larger than that of $f_{i_{1}}$, and the first $l-1$ coordinates are not from $I$. That implies that the number assigned to $B \cap S_{l}$ is strictly larger than the number assigned to $A \cap S_{l}$. (We use the fact that the numbers assigned to the elements of $\operatorname{conv}\left(g_{l}(P)\right)$ form an interval at this step.) Then $B \cap S_{l} \nsubseteq \nexists A \cap S_{l}$ (contradicting $B \subseteq A$ ) as the labeling of the elements of $S_{l}$ is done according to Lemma 3.1.

The following theorem provides a lower bound for $M_{j}(P, n)$. This bound, together with the upper bound of Theorem 2.2 proves Theorem 1.1, our main result.

Theorem 3.3. Let $P$ be a finite poset, $\varepsilon>0$ and $j \in\{1,2\}$. Then for all large enough $n$

$$
M_{j}(P, n) \geq \frac{1}{t_{j}(P)}\left(\begin{array}{c}
n \\
\lfloor n / 2\rfloor
\end{array}\right)(1-\varepsilon) .
$$

Proof. Choose $N, K$, and $f_{1}, f_{2}, \ldots, f_{K}: P \rightarrow B_{N}$ as in Lemma 3.2. (Use $\varepsilon^{\prime}=\frac{\varepsilon}{2}$ ). Consider the elements of $B_{N}$ as the subsets of a set $S$ of size $N$. Let $R$ be a set such that $S \subset R$ and $|R|=n$. Let $Q=R \backslash S$. Let

$$
\mathcal{Q}=\left\{T \subset Q\left|\left\lfloor\frac{n-N}{2}\right\rfloor-K \leq\right| T \mid \leq\left\lfloor\frac{n-N}{2}\right\rfloor-1\right\}
$$


If $n$ is large enough, then the following inequality is true:

$$
\sum_{i=1}^{K}\left(\begin{array}{c}
n-N \\
\left\lfloor\frac{n-N}{2}\right\rfloor-i
\end{array}\right) \geq K \cdot\left(\begin{array}{c}
n \\
\left\lfloor\frac{n-N}{2}\right\rfloor
\end{array}\right)\left(1-\frac{\varepsilon}{2}\right) .
$$

It can be verified easily by induction on $N$ that $2^{N}\left(\begin{array}{c}n-N \\ \left\lfloor\frac{n-N}{2}\right\rfloor\end{array}\right) \geq\left(\begin{array}{c}n \\ \left\lfloor\frac{n}{2}\right\rfloor\end{array}\right)$. Using this, we get that:

$$
|\mathcal{Q}| \geq K \cdot\left(\begin{array}{c}
n-N \\
\left\lfloor\frac{n-N}{2}\right\rfloor
\end{array}\right)\left(1-\frac{\varepsilon}{2}\right) \geq \frac{2^{N}\left(1-\frac{\varepsilon}{2}\right)}{t_{j}(P)} \cdot 2^{-N}\left(\begin{array}{c}
n \\
\left\lfloor\frac{n}{2}\right\rfloor
\end{array}\right)\left(1-\frac{\varepsilon}{2}\right) \geq \frac{1}{t_{j}(P)}\left(\begin{array}{c}
n \\
\lfloor n / 2\rfloor
\end{array}\right)(1-\varepsilon) .
$$

We define an embedding $f_{T}: P \rightarrow B_{R}$ (induced if $j=2$ ) for every $T \in \mathcal{Q}$ such that $\left\{f_{T}(P) \mid T \in \mathcal{Q}\right\}$ is an incomparable family. These embeddings map the elements of $P$ to subsets of the set $R=Q \cup S$. We will define them by determining $f_{T}(x) \cap Q$ and $f_{T}(x) \cap S$ for every $x \in P$. (The main idea is that we make the resulting copies of $P$ pairwise incomparable by defining them such that when $f_{T}(x) \cap Q$ is large, then $f_{T}(x) \cap S$ is small.) For any $x \in P$ let $f_{T}(x) \cap Q=T$ and $f_{T}(x) \cap S=f_{\left\lfloor\frac{n-N}{2}\right\rfloor-|T|}(x)$. Then $f_{T}$ is obviously an embedding (induced if $j=2$ ).

Now we check that the $\left\{f_{T}(P) \mid T \in \mathcal{Q}\right\}$ is an incomparable family. Let $T_{1}, T_{2} \in \mathcal{Q}$ be different sets. Assume that $A_{1} \in f_{T_{1}}(P), A_{2} \in f_{T_{2}}(P)$ and $A_{1} \subseteq A_{2}$. Then $T_{1}=A_{1} \cap Q \subseteq A_{2} \cap Q=T_{2}$. Since $T_{1} \neq T_{2}$, $\left|T_{1}\right|<\left|T_{2}\right|$ holds. Since $A_{1} \cap S \in f_{\left\lfloor\frac{n-N}{2}\right\rfloor-\left|T_{1}\right|}(P)$ and $A_{2} \cap S \in f_{\left\lfloor\frac{n-N}{2}\right\rfloor-\left|T_{2}\right|}(P)$, Lemma 3.2. (iii) implies $A_{1} \cap S \nsubseteq A_{2} \cap S$. It contradicts $A_{1} \subseteq A_{2}$, so the family is indeed incomparable.

We found at least $\frac{1}{t_{j}(P)}\left(\begin{array}{c}n \\ \lfloor n / 2\rfloor\end{array}\right)(1-\varepsilon)$ different embeddings (induced if $j=2$ ) of $P$ to $B_{R}$, where $|R|=n$, such that the resulting copies form an incomparable family. It proves the theorem.

\section{Remarks}

In this section we exactly determine the maximum number of incomparable copies for certain posets. The problem has already been solved for the chain posets (posets with pairwise related elements).

Theorem 4.1. (Griggs, Stahl, Trotter) [9] Let $P^{h+1}$ be the chain poset with $h+1$ elements. Then for all $n \geq h$

$$
M_{1}\left(P^{h+1}, n\right)=\left(\begin{array}{c}
n-h \\
\left\lfloor\frac{n-h}{2}\right\rfloor
\end{array}\right) .
$$

We include an alternative proof for the sake of completeness. The following theorem will be used.

Theorem 4.2. (Bollobás) [1] Let $\left(A_{i}, B_{i}\right)(1 \leq i \leq m)$ be a family of sets such that $A_{i} \cap B_{i}=\emptyset$ for all $1 \leq i \leq m$, and $A_{i} \cap B_{j} \neq \emptyset$ for all $i \neq j(1 \leq i, j \leq m)$. Then

$$
\sum_{i=1}^{m} \frac{1}{\left(\begin{array}{c}
\left|A_{i}\right|+\left|B_{i}\right| \\
\left|A_{i}\right|
\end{array}\right)} \leq 1 .
$$

Proof. (Theorem 4.1.) Consider an embedding of $P^{h+1}$ into $B_{n}$. Let its maximal and minimal elements embedded into $C_{i}$ and $D_{i}$ respectively. $C_{i} \supset D_{i}$ implies $\bar{C}_{i} \cap D_{i}=\emptyset$. On the other hand, choosing these sets for all $i=1, \ldots, m$, the incomparability conditions imply $\bar{C}_{i} \cap D_{j} \neq \emptyset$. The theorem of Bollobás can be applied for the pairs $\left(\bar{C}_{i}, D_{i}\right)$ :

$$
\sum_{i=1}^{m} \frac{1}{\left(\begin{array}{c}
\left|\bar{C}_{i}\right|+\left|B_{i}\right| \\
\left|\bar{C}_{i}\right|
\end{array}\right)} \leq 1 .
$$


The fact that $\left|C_{i}-D_{i}\right| \geq h$ results in $\left|\overline{C_{i}}\right|+\left|D_{i}\right| \leq n-h$. Therefore the left hand side of (19) can be bounded in the following way.

$$
\frac{m}{\left(\begin{array}{c}
n-h \\
\left\lfloor\frac{n-h}{2}\right\rfloor
\end{array}\right)}=\sum_{i=1}^{m} \frac{1}{\left(\begin{array}{c}
n-h \\
\left\lfloor\frac{n-h}{2}\right\rfloor
\end{array}\right)} \leq \sum_{i=1}^{m} \frac{1}{\left(\begin{array}{c}
\left|\bar{C}_{i}\right|+\left|B_{i}\right| \\
\left|\bar{C}_{i}\right|
\end{array}\right)} \leq 1
$$

It proves the upper bound in the theorem.

The lower bound can be seen by an easy construction. Let $G \subset\{h+1, h+2, \ldots, n\}$ be a subset of size $\left\lfloor\frac{n-h}{2}\right\rfloor$. Then $P^{h+1}$ can be embedded to the sets $G,\{1\} \cup G,\{1,2\} \cup G, \ldots,\{1,2, \ldots, h\} \cup G$. We have $\left(\begin{array}{c}n-h \\ \left\lfloor\frac{n-h}{2}\right\rfloor\end{array}\right)$ such embeddings and the resulting copies form an incomparable family. This proves the lower bound.

Definition Let $h(P)$ be the height of the poset $P$, that is the number of elements in a longest chain in $P$ minus 1. We say that $P$ is slim if it can be embedded into $B_{h(P)} . P$ is called thin if it has an induced embedding into $B_{h(P)}$.

Theorem 4.3. If $P$ is a slim poset of height $h=h(P)$, then

$$
M_{1}(P, n)=\left(\begin{array}{c}
n-h \\
\left\lfloor\frac{n-h}{2}\right\rfloor
\end{array}\right) .
$$

If $P$ is thin, then

$$
M_{1}(P, n)=M_{2}(P, n)=\left(\begin{array}{c}
n-h \\
\left\lfloor\frac{n-h}{2}\right\rfloor
\end{array}\right) .
$$

Proof. Since $P^{h+1}$ is a subposet of $P$,

$$
M_{2}(P, n) \leq M_{1}(P, n) \leq M_{1}\left(P^{h+1}, n\right) .
$$

Now consider $M_{1}\left(P^{h+1}, n\right)$ pairwise incomparable copies of $P^{h+1}$ in $B_{n}$ as defined in Theorem 4.1. Their convex hulls are isomorphic to $B_{h}$, so we can embed $P$ to them (in an induced way if $P$ is thin). It proves $M_{1}(P, n) \geq M_{1}\left(P^{h+1}, n\right)$ for slim posets, and $M_{2}(P, n) \geq M_{1}\left(P^{h+1}, n\right)$ for thin posets.

We already determined the value of $M_{1}\left(P^{h+1}, n\right)$ in Lemma 4.1 , so the proof is completed.

Of course Theorem 4.3 does not contradict Theorem 1.1, since $t_{1}(P)=2^{h}$ and

$$
\frac{1}{2^{h}}\left(\begin{array}{c}
n \\
\left\lfloor\frac{n}{2}\right\rfloor
\end{array}\right) \sim\left(\begin{array}{c}
n-h \\
\left\lfloor\frac{n-h}{2}\right\rfloor
\end{array}\right)
$$

The smallest non-slim poset is $V_{2}$ with three elements, $a, b, c$ and the relations $a<b, a<c$. Now we give a large set of incomparable copies for all $n$. Fix the parameter $i\left(1 \leq i \leq\left\lfloor\frac{n+2}{4}\right\rfloor\right)$. Choose an element

$$
F \in\left(\begin{array}{c}
{[n-2 i]} \\
\left\lceil\frac{n}{2}\right\rceil-2 i+1
\end{array}\right)
$$

Then the sets

$$
\begin{aligned}
& F \cup\{n-2 i+3, n-2 i+4, \ldots, n\}, \\
& F \cup\{n-2 i+3, n-2 i+4, \ldots, n\} \cup\{n-2 i+1\}, \\
& F \cup\{n-2 i+3, n-2 i+4, \ldots, n\} \cup\{n-2 i+2\}
\end{aligned}
$$


form an embedding of the poset $V_{2}$. Let $\mathcal{P}_{i}$ denote the set of all such copies. It is trivial that the copies in $\mathcal{P}_{i}$ are pairwise incomparable. But not much more difficult to check that two copies chosen from $\mathcal{P}_{i}$ and $\mathcal{P}_{j}\left(1 \leq i<j \leq\left\lfloor\frac{n+2}{4}\right\rfloor\right)$, respectively, are also incomparable. Therefore

$$
\bigcup_{i=1}^{\left\lfloor\frac{n+2}{4}\right\rfloor} \mathcal{P}_{i}
$$

is a collection of pairwise incomparable embeddings of $V_{2}$. We conjecture that this is the largest one.

\section{Conjecture}

$$
M_{1}\left(V_{2}, n\right)=\sum_{i=1}^{\left\lfloor\frac{n+2}{4}\right\rfloor}\left(\begin{array}{c}
n-2 i \\
\left\lceil\frac{n}{2}\right\rceil-2 i+1
\end{array}\right) .
$$

We are indebted to the two anonymous reviewers for their useful comments.

\section{References}

[1] B. Bollobás, On generalized graphs, Acta Math. Acad. Sci. Hungar. 16 (1965) 447-452.

[2] B. Bukh, Set families with a forbidden subposet, Electronic J. of Combinatorics 16 (2009) R142, $11 \mathrm{p}$.

[3] H.-B. Chen and W.-T. Li, A Note on the Largest Size of Families of Sets with a Forbidden Poset, to appear in Order (2013)

[4] A. De Bonis and G. O. H. Katona, Largest families without an r-fork, Order 24 (2007) 181-191.

[5] A. P. Dove, J. R. Griggs, Packing Posets in the Boolean Lattice, preprint

[6] P. Erdős, On a lemma of Littlewood and Offord, Bull. Amer. Math. Soc. 51 (1945) 898-902.

[7] J. R. Griggs, W.-T. Li and L. Lu, Diamond-free families, J. Combinatorial Theory (Ser A) 119 (2012) 310-322.

[8] J. R. Griggs and L. Lu, On families of subsets with a forbidden subposet, Combinatorics, Probability, and Computing 18 (2009) 731-748.

[9] J. R. Griggs, J. Stahl, W. T. Trotter Jr., A Sperner Theorem on Unrelated Chains of Subsets, J. Combinatorial Theory (Ser. A) 36 (1984) 124-127.

[10] G. O. H. Katona and T. G. Tarján, Extremal problems with excluded subgraphs in the n-cube, Lecture Notes in Math. 1018 (1981) 84-93.

[11] L. Kramer, R. R. Martin and M. Young, On diamond-free subposets of the Boolean lattice, J. of Combinatorial Theory, (Ser. A) 120(3) (2012), 545-560. 